\title{
Cold urticaria with risk of anaphylaxis treated with omalizumab. A case report
}

\section{Urticaria por frío y riesgo de anafilaxia tratada con omalizumab. A propósito de un caso}

\author{
Tonatiuh Ramsés Bedolla-Pulido, ${ }^{1}$ Juan Mariscal-Castro, ${ }^{1}$ Tania González-Mendoza, ${ }^{1}$ \\ Jaime Morales-Romero, ${ }^{2}$ Martín Bedolla-Barajas ${ }^{1}$
}

\begin{abstract}
Background: Omalizumab, which is a monoclonal anti-IgE antibody, has recently been used as an option in the treatment of inducible urticaria.

Case report: We describe the case of a 46-year-old woman who was referred to the Department of Allergy and Immunology of "Hospital Civil de Guadalajara, Dr. Juan I. Menchaca" due to a history of hives, body itching, changes in the color of the skin after exposure to water, and chest tightness after the intake of cold beverages; therefore, she used to limit her outdoor activities and personal hygiene. We conducted challenge tests with heat, soaked towels, treadmill walks, and dermographism; which were negative. The ice cube test was positive. To establish the speed of wheal formation, we established intervals of exposure to cold of one, three, five, and ten minutes; a positive result was obtained from the third minute. Due to the poor response to the drug treatment and to measures to avoid the cold, as well as to the poor quality of life, the high risk of anaphylaxis, and the advent of winter season, omalizumab was administered at monthly doses of $150 \mathrm{mg}$ during the winter season. After the first dose, there were no reports of episodes of hives in areas exposed to cold; the ice cube test was negative before the second dose and in the following months, and the patient was able to ingest cold beverages and cold food. There were no adverse reactions that could be attributable to the use of omalizumab. Three years after the first dose, the patient was still asymptomatic.

Conclusion: The described case is one of the first cases of cold urticaria with risk of anaphylaxis with a positive response to omalizumab, which was reflected in symptom control and the improvement in the quality of life.
\end{abstract}

Key words: Cold urticaria; Anaphylaxis; Omalizumab

Este artículo debe citarse como: Bedolla-Pulido TR, Mariscal-Castro J, González-Mendoza T, Morales-Romero J, Bedolla-Barajas M. Urticaria por frío y riesgo de anafilaxia tratada con omalizumab. A propósito de un caso. Rev Alerg Mex. 2020;67(4):408-412

ORCID

Tonatiuh Ramses Bedolla-Pulido, 0000-0002-5292-0728; Juan Mariscal-Castro, 0000-0002-7907-5309; Tania González-Mendoza, 0000-0003-4121-0293; Jaime Morales-Romero, 0000-0002-1492-1797; Martín Bedolla-Barajas, 0000-0003-4915-1582 


\section{Resumen}

Antecedentes: Omalizumab, un anticuerpo monoclonal anti-IgE, recientemente es utilizado como una opción en el tratamiento de la urticaria inducible.

Caso clínico: Describimos el caso de una mujer de 46 años referida al Servicio de Alergia e Inmunología del Hospital Civil de Guadalajara Dr. Juan I. Menchaca, por historia de urticaria, picazón en el cuerpo, cambios en el color de la piel tras la exposición al agua y opresión en el pecho posterior el consumo de bebidas frías; en consecuencia, ella limitaba sus actividades al aire libre y de higiene personal. Se realizaron pruebas de desafío con calor, toalla húmeda, caminata en banda sinfín y dermografismo, las cuales fueron negativas; la prueba del cubo de hielo fue positiva. Para establecer la velocidad de la formación de la roncha establecimos intervalos de exposición al frío durante uno, tres, cinco y 10 minutos; se obtuvo un resultado positivo desde el tercer minuto. Debido a la mala respuesta al tratamiento farmacológicos y a las medidas para evitar el frío, así como por la mala calidad de vida, el alto riesgo de anafilaxis y el advenimiento de la temporada de invierno, se administraron $150 \mathrm{mg} / \mathrm{mes}$ de omalizumab durante la temporada de invierno. Después de la primera dosis no se notificaron episodios de urticaria en zonas expuestas al frío; la prueba del cubo de hielo antes de la segunda dosis y en los meses siguientes fue negativa y la paciente pudo ingerir bebidas y alimentos fríos. No se produjeron reacciones adversas atribuibles al uso de omalizumab. Tres años después de la primera dosis, la paciente permanecía asintomática.

Conclusión: El caso descrito constituye uno de los primeros de urticaria inducida por el frío con riesgo de anafilaxia con respuesta positiva a omalizumab, lo que se reflejó en el control de los síntomas y la mejoría en la calidad de vida.

Palabras clave: Urticaria inducida por el frío; Anafilaxia; Omalizumab

${ }^{1}$ Hospital Civil de Guadalajara Dr. Juan I. Menchaca, Servicio de Alergia e Inmunología Clínica, Jalisco, México ${ }^{2}$ Universidad Veracruzana, Instituto de Salud Pública, Veracruz, México
Correspondencia: Martín Bedolla-Barajas. drmbedbar@gmail.com

Recibido: 2020-04-19

Aceptado: 2020-07-14

DOI: $10.29262 /$ ram.v67i4.744
Abreviaturas y siglas
ColdU, cold urticaria
CU-Q2oL, cuestionario de calidad de vida

CVF, capacidad vital forzada

\section{Antecedentes}

Después del dermografismo, la urticaria por frío (Col$\mathrm{dU}$, cold urticaria) es la segunda urticaria inducible más frecuente. ${ }^{1}$ Wanderer et al. categorizaron a la ColdU de acuerdo con la gravedad de los síntomas: ${ }^{2}$

- Tipo I, cuando la urticaria o angioedema están localizados.

- Tipo II, cuando la urticaria o angioedema están generalizados, pero no existen síntomas de hipotensión o respiratorios.
- Tipo III, cuando la reacción es sistémica, caracterizada por episodios de distrés respiratorio o hipotensión, este tipo de reacción en particular ocurre con mayor frecuencia en pacientes con prueba de tiempo de estimulación con frío $\geq 3 \mathrm{~min}$.

Por otra parte, un poco más de la tercera parte de los pacientes con ColdU tiene riesgo de desarrollar anafilaxia por este mismo motivo. ${ }^{3}$

Actualmente, las guías internacionales recomiendan el uso de antihistamínicos $\mathrm{H} 1$ de segunda 


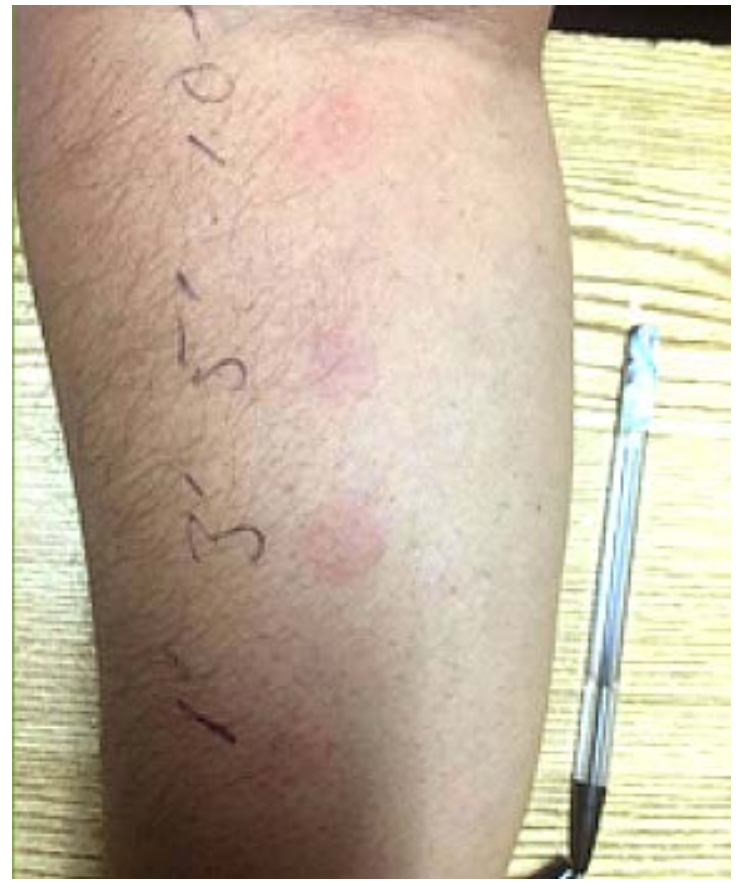

Figura 1. Erupciones y eritema después de la prueba del cubo de hielo (uno, tres, cinco y 10 minutos después de la exposición).

generación como primera línea terapéutica de la urticaria; pero cuando la respuesta no es favorable aun con dosis altas, entonces se sugiere el uso de omalizumab. ${ }^{4}$

Si bien se ha demostrado el beneficio de omalizumab en el tratamiento de ColdU, su utilidad en ColdU clase III sigue siendo limitada. De tal forma, el objetivo de este trabajo fue presentar el caso de una paciente con ColdU refractaria al tratamiento inicial y con riesgo de anafilaxia quien fue tratada con omalizumab.

\section{Caso clínico}

Mujer de 46 años de origen mexicano con historia personal de asma, sin ocupación remunerada y dedicada a las labores del hogar. Al momento de la primera evaluación, su padecimiento tenía aproximadamente dos meses de evolución.

Las molestias consistían en prurito, ronchas y cambios de la coloración de la piel, sobre todo en las zonas de contacto con el agua; la paciente experimentaba opresión faríngea después del consumo de alimentos o líquidos fríos; limitó su aseo personal y las actividades al aire libre por temor a empeorar. En general, no había sido satisfactoria la respuesta al uso de antihistamínicos, antileucotrienos y corticosteroides sistémicos, a diferentes dosis y combinaciones.

De acuerdo con la historia de síntomas al contacto con el agua, se realizó una prueba con lienzo húmedo; después de 10 minutos la paciente manifestó prurito y se observaron ronchas en la zona que había tenido contacto con el agua. En la prueba de caminata en una banda sinfín, la paciente manifestó prurito corporal; además, se observaron habones en la zona de la " $v$ " del escote, en los brazos y antebrazos, abdomen y región lumbar. Dado que ambas pruebas se hicieron en habitaciones climatizadas con aire frío, las pruebas se repitieron en un ambiente cálido, donde las pruebas fueron negativas. La prueba de reto con calor fue negativa; tampoco hubo dermografismo. Notoriamente, la prueba del cubo de hielo fue positiva. Con la finalidad de determinar la velocidad de formación de la erupción, la prueba del cubo de hielo se repitió, manteniendo la exposición al frío durante uno, tres, cinco y $10 \mathrm{mi}$ nutos. El resultado fue positivo a partir del tercer minuto (figura 1).

Ante la historia de pobre respuesta al tratamiento con medicamentos y medidas de evitación del frío, así como por la mala calidad de vida, el riesgo alto de anafilaxia y el advenimiento de la época invernal en la región de residencia de la paciente, se tomó la decisión de administrar $150 \mathrm{mg}$ de omalizumab cada mes durante el invierno (de noviembre a febrero).

Después de la primera dosis de omalizumab, la paciente dejó de presentar episodios de urticaria en las zonas de exposición al frío. El día de la segunda aplicación y seis meses después de la cuarta y última dosis, nuevamente se realizó la prueba de cubo de hielo, la cual fue negativa. Para entonces, la paciente ya había logrado ingerir bebidas y alimentos fríos sin presentar molestias. Antes de la primera dosis de omalizumab, la puntuación del cuestionario de calidad de vida (CU-Q2oL) ${ }^{5}$ fue de 25; antes de la segunda dosis, de 5.4 puntos; y seis meses después de la última dosis, de 7.6 puntos. Durante el periodo de observación no se registraron reacciones adversas atribuibles al uso de omalizumab.

Las pruebas de función respiratoria mostraron capacidad vital forzada (CVF) de $93 \%$ del valor 
predicho, volumen espiratorio forzado el primer segundo (FEV1) de $93.8 \%$ del valor predicho y una relación FEV1/CVF de $99 \%$ del valor predicho. Los parámetros de la biometría hemática se encontraron dentro de los rangos de referencia. Las pruebas cutáneas para aeroalérgenos de la región no fueron valorables, pues la temperatura de la gota que contiene al alérgeno $\left(\right.$ de 2 a $8{ }^{\circ} \mathrm{C}$ ) fue un factor suficiente para despertar la formación de una erupción.

Después de transcurrido un año de la administración de la primera dosis de omalizumab, la paciente permanecía asintomática.

\section{Discusión}

Presentamos el caso de una mujer con ColdU, con riesgo elevado de presentar anafilaxia, tratada exitosamente de manera temprana con omalizumab.

En general, hasta una tercera parte de los pacientes con ColdU manifiestan anafilaxia como parte del espectro de síntomas de este trastorno. ${ }^{6}$ A través de la prueba del cubo de hielo es posible identificar los casos con riesgo de desarrollar síntomas sistémicos o anafilaxia relacionada con la exposición al frío; también es viable anticipar el tratamiento y disminuir los riesgos de complicaciones.

En la paciente que aquí se presenta se realizaron pruebas de provocación para diferenciar entre los diferentes subtipos de urticaria, según lo recomiendan las guías de diagnóstico de las urticarias inducibles; ${ }^{4}$ fue así como se descartó la urticaria colinérgica, la acuagénica, la fáctica y por calor y, finalmente, se confirmó el diagnóstico de ColdU tipo III.

Dos aspectos importantes por discutir están relacionados con la dosis necesaria de omalizumab para lograr el control de la ColdU, así como el momento en el cual debe administrarse. Respecto al primero, en nuestra paciente observamos una respuesta clínica rápida una semana después de utilizar la primera dosis de $150 \mathrm{mg}$. La paciente pudo ingerir alimentos fríos y un mes después la prueba de cubo de hielo fue negativa. Al revisar la literatura encontramos que el primer caso de ColdU tratado con omalizumab fue una niña con síntomas sistémicos después de nadar en el mar; la administración de $375 \mathrm{mg}$ de omalizumab cada dos semanas fue suficiente para lograr la resolución completa de la urticaria. ${ }^{7}$ Recientemente, un ensayo clínico mostró que $150 \mathrm{mg}$ o $300 \mathrm{mg}$ de omalizumab es igualmente útil para lograr tasas altas de respuestas completas y parciales. ${ }^{8}$ En cuanto al segundo punto, las guías internacionales aconsejan el omalizumab como tercera línea de tratamiento; sin embargo, la experiencia aportada en el caso que describimos sugiere que probablemente su uso temprano pudiera contribuir a la remisión de la ColdU. Dado el bajo nivel de evidencia que suele aportar la presentación de casos clínicos únicos, ${ }^{9}$ hacen falta estudios encaminados a responder esta interrogante.

Ahora bien, cabe preguntarse cuál de las siguientes fue la causa de la remisión del cuadro de la ColdU en la paciente:

\section{- ¿Omalizumab?}

- ¿Se trató de una coincidencia producto del azar (remisión espontánea)?

Sin duda, se trata de una pregunta de investigación. Después de un año completo de seguimiento, la paciente se mantenía asintomática, con buena calidad de vida y sin molestias de asma. Como se mencionó, el nivel de evidencia aportado por la descripción de un solo caso dificulta precisar si el omalizumab fue responsable de la remisión de la ColdU. En un estudio de cohorte se encontró una tasa de resolución espontánea de la ColdU de 18 y $24 \%$ a los cinco y 10 años, respectivamente; notoriamente, la historia personal de enfermedad atópica estuvo relacionada con menor probabilidad de resolución. ${ }^{6}$

Aunque hasta el momento no han sido claramente definidos los mecanismos por los cuales omalizumab es efectivo en el tratamiento de la ColdU, se ha propuesto la existencia de una inmunoglobulina E específica dirigida contra alguna proteína derivada de la piel, la cual se ve activada por la exposición al frío. ${ }^{8}$

\section{Conclusión}

Informamos uno de los primeros casos de ColdU con riesgo de anafilaxia, que mostró respuesta positiva al uso de omalizumab, la cual se tradujo en un control prolongado de los síntomas y en una mejor calidad de vida. 


\section{Referencias}

1. Siebenhaar F, Weller K, Mlynek A, Magerl M, Altrichter S, Vieira-Dos Santos R, et al. Acquired cold urticaria: clinical picture and update on diagnosis and treatment. Clin Exp Dermatol. 2007;32(3):241-245. DOI: 10.1111/j.1365-2230.2007.02376.x.

2. Wanderer AA. Cold urticaria syndromes: historical background, diagnostic classification, clinical and laboratory characteristics, pathogenesis, and management. J Allergy Clin Immunol. 1990;85(6):965-981. DOI: 10.1016/0091-6749(90)90037-5

3. Alangari AA, Twarog FJ, Shih MC, Schneider LC. Clinical features and anaphylaxis in children with cold urticaria. Pediatrics. 2004;113(4):313-317. DOI: 10.1542/peds.113.4.e313

4. Zuberbier T, Aberer W, Asero R, Abdul Latiff AH, Baker D, Ballmer-Weber B, et al. The EAACI/GA²LEN/ EDF/WAO guideline for the definition, classification, diagnosis and management of urticaria. Allergy. 2018;73(7):1393-1414. DOI: 10.1111/all.1339

5. Aguilar-Hinojosa NK, Segura-Méndez NH, Lugo-Reyes SO. Correlación de la gravedad de urticaria crónica y la calidad de vida. Rev Alerg Mex. 2012;59(4):180-186.

6. Jain SV, Mullins RJ. Cold urticaria: a 20-year follow-up study. J Eur Acad Dermatol Venereol. 2016;30(12):2066-2071. DOI: 10.1111/jdv.13841

7. Boyce JA. Successful treatment of cold-induced urticaria/anaphylaxis with anti-lgE. J Allergy Clin Immunol. 2006;117(6):1415-1418. DOI: 10.1016/j.jaci.2006.04.003.

8. Metz M, Schütz A, Weller K, Gorczyza M, Zimmer S, Staubach P, et al. Omalizumab is effective in cold urticaria-results of a randomized placebo-controlled trial. J Allergy Clin Immunol. 2017;140(3):864-867. DOI: 10.1016/j.jaci.2017.01.043

9. Guyatt GH, Haynes RB, Jaeschke RZ, Cook DJ, Green L, Naylor CD, et al. User's guides to the medical literature: XXV. Evidence-based medicine: principles for applying the user's guides to patient care. Evidence-Based Medicine Working Group. JAMA. 2000;284(10):1290-1296. DOI: 10.1001/ jama.284.10.1290 